\title{
Interkulturelle Mediävistik als Projekt
}

\author{
Perspektiven und Potentiale vormoderner Transkulturalität: \\ Herzog Ernst (B) und Die Geschichten aus 1001 Nacht
}

Dina Авoul Fotoun Hussein Salama ${ }^{1}$

\begin{abstract}
This study proposes a new concept of Intercultural Medieval Studies, following Heimböckel's and Weinberg's notion of interculturality as a project. It conceptualizes cultural diversity as a process of communicative entanglements, connections, experiences etc. that have always already transgressed cultural boundaries. Such cultural fusions influence identity constructions and cultural life in general, and in particular, they shape premodern literature in multiple ways. After discussing various concepts of culture, with special emphasis on the difference between interculturality and transculturality, which this study regards as complementary terms, the study explores the application of inter-and transculturality in academic teaching as well as in research. It suggests topics and literary works that can be associated with different concepts of culture, and exemplifies one such association in a case study that compares the description of the East in the anonymous novel Herzog Ernst (B) with similar motives and narratives in The Stories of the 1001 Nights, putting emphasis on moments of astonishment as a »liminal phenomenon « (Heimböckel/Weinberg), and on the affects that determine cultural contact and interaction.
\end{abstract}

Title: Intercultural Medieval Studies as a Project: Perspectives and Potentials of Premodern Transculturality

Keywords: intercultural experiences; transculturality; medieval studies; Herzog Ernst (B); The 1001 Nights

\section{VorÜBerlegungen ZUM KuLtURBegrifF}

Versuche, einen Konsens für den Kulturbegriff zu formulieren - Gisela Trommsdorf konstatiert über hundert verschiedene Definitionsversuche (vgl. 2000: 388) -, münden meist darin, »schwer zu beschreibende komplexe Phänomene mit einem anspruchsvollen Begriff zu versehen und das >label mit der Erklärung des Phänomens zu verwechseln« (ebd.), oder sogar darin, die Begriffsbildung selbst zu hinterfragen (vgl. Eibach/Opitz-Belakhal/Juneja 2012: Abschnitt 2). Zu beobachten ist, dass der Begriff Kultur als Untersuchungskategorie in der Forschung zunehmend zwischen gegebenem Gegenstand und wandelbarem Prozess oszilliert und meist ein Sowohl-als-auch impliziert und sich

1 | Universität Kairo. 
so von der Vorstellung von geschlossenen Einheiten, »Kugeln« (Herder 1891: 509), entfernt.

In Abhängigkeit von Subjekten, die als persönliche Identitäten »mit dem ständigen Wechsel zwischen den einzelnen Wertsphären, Subsystemen oder sozialen Kreisen « zurechtkommen, deren Intensität sich »durch die Allgegenwart von neuen Medien, globalen Mobilitätsschüben und dem Entstehen neuer ethnischer und religiöser Identifikationsmöglichkeiten verschärft« (Hahn/Willems 1999: Klappentext), wird Kultur, die diesen vielseitigen und mehrdimensionalen Wandel durchläuft, sowohl als Konstante als auch als Prozess begriffen. Im Rahmen von entwicklungspsychologischen Theorien wird Kultur als

Ergebnis von Interaktionen und von Sozialisations- und Transmissionsprozessen, und zwar sowohl in der Entwicklung des einzelnen als auch im soziokulturellen Kontext, der sich über die Zeit verändern kann, verstanden. Damit wird der Blick auf den Prozeß der Wechselwirkungen zwischen Individuum und Kultur gerichtet. (Trommsdorf/Friedlmeier 2004: 380f.)

Besonders hervorzuheben sind die Versuche der historischen Wissenschaften, speziell im Rahmen der kollaborativ verfassten Studie des Netzwerks >Transkultureller Verflechtungen< (vgl. Mersch 2016) des Centres for Transcultural Studies der Ruprecht-Karls-Universität Heidelberg, die vielfältigen und mehrdimensionalen Prozesse der Wandlungen von Kultur, der Transkulturalität (vgl. Welsch 2010), aus mediävistischer Perspektive mit visualisierenden Beschreibungen wie Verflechtungen, Entflechtungen und Geflecht zu verbalisieren. In diesem Sinne schlägt Margit Mersch in ihrer Studie Transkulturalität, Verflechtung, Hybridisierung - >neue< epistemologische Modelle in der Mittelalterforschung für die Konzeptualisierungen der dynamischen Multidimensionalität, der dezentralen Multidirektionalität und Vielschichtigkeit sowohl die Metapher des >Rhizoms < als auch die des >Palimpsests < vor (vgl. Mersch 2016: 248-250):

Das Modell des Rhizoms verweist auf geflechtartige Beziehungen auf unterschiedlichen Ebenen und in unterschiedlichen Dimensionen und Richtungen, auf Beziehungsgeflechte, die keine klaren inneren oder äußeren Abgrenzungen erkennen lassen. Wie gut sich dieses epistemologische Modell für historische Analysen eignet, um die Multidimensionalität und Multidirektionalität der Beziehungen auszudrücken und zugleich anzudeuten, dass diese historisch gewachsen sind und sich fortgesetzt weiterentwickeln, zeigt der Erfolg der Begriffe Verflechtung und Geflecht, die sich parallel zum Terminus Transkulturalität allgemein durchgesetzt haben, freilich ohne die komplizierte Rhizom-Theorie von Deleuze und Guattari explizit zu adaptieren. Die metaphorische Verwendung der Begriffe Rhizom oder rhizomatisches Geflecht ermöglicht dabei auch, neben der sozialen Praxis der Akteure und ihren Konzeptualisierungen die unbewussten bzw. nichtintentionalen Prozesse und verdeckte Beziehungen in den Blick zu bekommen. Wie ein Pilzgeflecht aus weitläufigen, verzweigten unterirdischen Verbindungen und vereinzelten, lokal begrenzten oberirdischen Fruchtkörpern zeigen sich in der mittelalterlichen Kulturgeschichte an geographisch und historisch weit voneinander 
entfernten oder/ und politisch getrennten Orten einzelne einander ähnliche oder aufeinander verweisende kulturelle Manifestationen (etwa architektonisches Formengut), die auf die Existenz verborgener, mittelbarer Zusammenhänge hinweisen. Letztere müssten rätselhaft bleiben, wenn man nur die offensichtlichen linearen Verbindungen gelten lassen wollte, anstatt die potentielle Vielgestaltigkeit historischer Beziehungen zu bedenken und offen für mögliche Richtungen, Richtungswechsel und die zeitliche Tiefe von Verflechtungen wie auch gegenüber scheinbar paradoxen Entwicklungen zu sein. (Ebd.: 249)

In meinen gedanklichen Ausführungen möchte ich diese Verflechtungen und Valenzen mit der Durchlässigkeit von (flüssigen) Farben vergleichen, die individuelle Melangen ergeben. Wissensbestände, zu denen Verhaltensmuster, Motive, aber auch Narrative u.a. zählen, formen sich hierbei jedes Mal aufs Neue $\mathrm{zu}>$ Trans-Elementen<. Mit Trans-Elementen möchte ich die Segmente bezeichnen, die Teil eines Ganzen sind und sich im Ganzen zu einer einzigartigen Melange auflösen und zugleich Teil eines anderen Ganzen oder Teils sein können. Diese mehrdimensionalen Optionen von Farb-Zugehörigkeiten bilden die Momente, die sich aus der speziellen Mischung als Kultur bzw. Individualität, aber auch unter dem Aspekt der prozessualen und dynamischen Durchlässigkeit und Vielfalt als Transkulturalität auszeichnet. Dies impliziert einen offenen Kulturbegriff, der in sich Heterogenität mit Homogenität vereint. Vor dem Hintergrund dieser Vielseitigkeit und Polyvalenz konstituieren Individuen eine transkulturelle Identität, die in sich aufgrund und als Folge diverser Kollektivzugehörigkeiten $^{2}$ sowohl partizipative als auch funktionale Verbindungslinien (vgl. Hahn 2000) verknüpft.

$\mathrm{Zu}$ vergleichen ist dies mit einem Gemälde, dessen Farben aus der Distanz einen bestimmten Ton erkennen lassen, der allerdings, aus unmittelbarer Nähe betrachtet, aus vielen mikroskopischen unterschiedlichen Mischungen von Farbpigmenten besteht, von denen die identischen als ähnlich und die andersfarbigen als different erkannt werden können. In Analogie dazu sind die inter- und transkulturellen Projekte zu verstehen, die, über Transformationsprozesse reflektierend, sowohl >Ähnlichkeit< (vgl. Bhatti 2015) als auch Momente der Grenzziehung und >Differenz « (vgl. Hofmann 2006; Heimböckel/Weinberg 2014 u.a.) fokussieren. Im Hinblick auf diese prozessualen, unendlichen Wandlungen erscheint eine verabsolutierende kategorische Zuordnung und Vereindeutigung dieser Segmente als schwierig, fast als unmöglich. Daher gehe ich in meinen Ausführungen von dem Gedanken einer Kulturmelange aus, die auf der Reziprozität fließender kultureller Prozesse, von Wandlungen sowie der »Wanderschaft durch Zeiten, Räume und Medien mit ihrer Vielzahl von Schwellenübertritten«

2 | Claus Leggewie u. Dariuš Zifonun (vgl. 2010: 24-27) fassen die prozessualen und institutionellen Mechanismen des Ambivalenzmanagements unter den Bedingungen von Interkulturalität anhand von sieben Punkten zusammen. 
(Herweg 2017: 16) basiert und die mit jeder Erkundung - von außen und von innen - stets neue Töne ergibt. ${ }^{3}$

In Analogie zur Inter- und Transtextualität, wonach Texte aus anderen Texten entstehen und es keine Autonomie der Texte gibt, liegt auch jeder Kultur selbst ein Text (vgl. Geertz 1994) bzw. liegen der Kultur andere Kulturen bzw. Subkulturen zugrunde, die sich in einem universalen Prozess in stetigem Wandel befinden. Im Hinblick auf diesen Synkretismus möchte ich einen offenen Kulturbegriff ableiten, der als Ergebnis von unendlichen inter- als auch transkulturellen Prozesssen zu verstehen ist.

Mit diesen Vorstellungen möchte ich meinen Vorschlag für die Möglichkeiten der Systematisierung einer Interkulturellen Mediävistik einleiten, die von einer Transkulturalität der Kulturen ausgeht und diese in ihrem Konzeptualisierungsversuch einbindet. Bei diesem Versuch geht es darum, den von Heinz Sieburg (vgl. 2015/2016: 399) eingeschlagenen Weg zur Systematisierung einer Interkulturellen Mediävistik, in der Transkulturalität ebenfalls stets mitgedacht wird, weiter auszubauen und über die Einbindung internationaler Lehr- und Forschungserfahrungen, insbesondere der ägyptischen Mediävistik, zu erweitern. In Anlehnung an die Ausführungen Dieter Heimböckels und Manfred Weinbergs (vgl. 2014) wird Interkulturalität als Projekt konzipiert, wie weiter auszuführen sein wird. Zuvor seien vor dem Hintergrund der Mediävistik noch kurz einige Reflexionen über das Verhältnis der Begrifflichkeiten von Inter- und Transkulturalität dargelegt, die hier nicht in Konkurrenz zueinander, sondern in ihrer Komplementarität angesehen werden (vgl. Iljassova-Morger 2009: 44).

\section{Reflexionen ZUM INTER der Kulturen UND IHRER TRANSKULTURALITÄT}

Nach Michael Hofmann bezieht sich >Interkulturalität < auf die Konstellation der Begegnung zweier oder mehrerer Subjekte. Im Rahmen der Anwendung der Interkulturellen Literaturwissenschaft hebt Hofmann (vgl. 2006: 12) den prozesshaften und dialogischen Charakter des Kulturbegriffs heraus, der gemäß Ortrud Gutjahr

3 IIm Rahmen seiner Ausführungen zum "präkolonialen Literaturtransfer" stellt Mathias Herweg (vgl. 2017: 15f.) die von inm vorgestellen literarischen "Phänomene" eines Vernetzungsprozesses dar, den er mit dem Schlagwort des ex oriente lux verbindet. Herweg begreift diese Quellen als Beispiele "für die vitale Vernetzung der westöstlichen Literaturen in (Spät-)Antike, Mittelalter und Frühneuzeit (jenen Epochen, die ich wertungsfrei als ,Vormoderne ( fasse). Sie sind, wenn man so will, 'Migrationsliteratur in einem ganz eigenen Sinn. Wie sich die Migration in jedem Einzelfall vollzog und niederschlug, dies zu klären bleibt eine Zukunftsaufgabe, die so weit über die Germanisitik hinausgeht, wie die beteiligten Sprachen es fordern.“ (Ebd.: 16) 
an der Selbstthematisierungsfähigkeit von Gesellschaft ansetzt und die kontextbezogene Veränderung von Bedeutungen und Menschen, die sich situativ und multipel verorten, untersucht. In diesem Oszillieren zwischen unterschiedlichen Handlungsorientierungen kommt es zur Auflösung und Neuschaffungen von Grenzziehungen, Machtund Gewaltverhältnissen und Geschlechterrollen. So wird mit dem Begriff Interkulturalität eine Grenzüberschreitung in den Blick genommen, bei der weder ein wie auch immer gefasstes Innerhalb oder Außerhalb der Grenze noch die Grenze zum eigentlichen Untersuchungsgegenstand wird, sondern das Inter selbst. Mithin geht es um die Funktionsweise von Differenzbestimmungen, die Kulturationsprozesse abstützen, verändern oder neu in Gang setzen. (Gutjahr 2002: 352)

Beschreibt Steven D. Martinson den Begriff der Transkulturalität als eine »wechselwirkende[n] Interaktion verschiedener kultureller Elemente« und als »Transformation von zwei oder mehr Kulturen in ausgeweiteten Identitäten, während diese Kulturen ihre authentischen Grundformen behalten « (Martinson 2008: 75), so begreift Albrecht Classen Transkulturalität als Reaktion der Vertreter der einen Kultur auf Elemente einer anderen, selbst wenn dies auf negative Weise erfolge: »Transculturality begins when representatives of one culture react to elements of another culture, even when this happens in negative terms at first.« (Classen 2015: 682)

Vor diesem Hintergrund möchte ich unter einer interkulturellen Analyse in der Literaturwissenschaft die Erkundung des spezifischen und >bedeutsamen (vgl. Dembeck 2017: 10) Moments der Begegnung auf inhaltlicher Ebene verstehen. Genau auf diesen Punkt kann sich die interkulturelle Analyse konzentrieren, als ein Verfahren, das sich auf den Moment der Begegnung fokussiert und sich mit den Modi der vielfachen und reziproken Erkundung beschäftigt, die als sich wiederholender Verflechtungs- und Entflechtungsprozess die Transkulturalität von Identitäten und ihren Werken ergibt.

Kulturen (und mit innen Interkulturalität) kann es nur geben, wenn sich ein Punkt angeben läßt, an dem die eine Kultur aufhört und die nächste anfängt. Solche Differenzierbarkeit von Kulturen erhält im topographischen Modell der Grenze ihre allegorische Zuschreibung. Doch auch wenn diese Grenze die Bedingung der Möglichkeit von Interkulturalität bildet, heißt dies nicht, daß interkulturelle Begegnungen an und auf dieser Grenze stattfinden. Die Begegnung zweier Kulturen findet vielmehr statt, wenn eine Kultur in das abgegrenzte Terrain der anderen einbricht. Interkulturalität setzt somit beides voraus: eine Grenze zwischen den Kulturen - und ihre Überschreitung. (Rieger/ Schahadat/Weinberg 1999: 11)

Dazu führen Heimböckel/Weinberg (2014: 130) aus: »Auf das im Konzept der Interkulturalität aufgerufene >Zwischen < fokussierend, heißt dies aber, dass es einerseits nicht die Zwischenstellung der Grenze selbst meinen kann, andererseits aber auch nicht unabhängig von der Grenze/von Grenzen zu denken ist.«

Vor dem Modell eines offenen, prozessualen und verflüssigten Kulturbegriffs erfolgt über jede Erkundung und Neuschaffung von Grenzziehung eine 
Transformation, die als unendlicher Prozess der Verwandlung letztendlich die Transkulturalität des Individuums konstituiert und immer wieder von Neuem sichtbar macht. Die Summe interkulturell und akkumulativ äußerst verflochtener Prozesse der interkulturellen Begegnung (Vermischung und Aneignung, aber auch Distanzierung und Ablehnung/kritische Reflexion u.a.) ergibt transkulturelles Wissen, das folglich mitunter in die künstlerischen Schaffensprozesse dieses Gemäldes einfließt, so dass die Grenzen zwischen den mit- und ineinander vermischten kulturellen Farben verfließen und sich keine eindeutige Grundfarbe fixieren lässt.

Betrachtet man - so mein Vorschlag - Transkulturalität als Produkt interkultureller Begegnungen, wobei unter Begegnung und Erkundung eine unendliche Reihe von Formen und Möglichkeiten der Aneignung kognitiver, physischer, psychischer, rechtlicher, sprachlicher Elemente bzw. Nuancen u.a. zu verstehen ist, so ergibt sich aus diesen Prozessen ein Subjekt, das als Teilnehmer an verschiedenen Aktionsräumen bzw. Subkulturen auf individuelle Weise als transkulturelle Identität konstituiert ist. Die Teilhabe an verschiedenen kollektiven Gruppen kann zugleich als Indikator für die Fähigkeit zur Integration - z.B. durch Bewältigung von Konflikten, Überstehen von Abenteuern, Assimilation, aber auch und vor allem durch räumliche Vernetzung und Konnektivität sowie Aushandlungs- und Verhandlungsgeschick - dienen und diese garantieren. ${ }^{4}$ Ludger Pries stellt in seinen Ausführungen im Rahmen der Arbeitsmigration den Transmigranten als neuen Wanderungstypus vor, den er von dem Einwanderer, dem Gastarbeiter und den in der Diaspora herausgebildeten Minderheiten differenziert:

Hierbei wird das Verhältnis zwischen Herkunfts- und Ankunftsregion durch die Herausbildung von auf Dauer angelegten Transnationalen Sozialen Räumen gestaltet. Diese sozialen Räume fallen nicht eindeutig mit einheitlichen Flächenräumen zusammen, wie es im Falle der 'Auswanderer (Ankunftsland) und der rückkehrenden ,Gastarbeiter (Herkunftsland) ist. Sie sind auch nicht einfach ein flächenräumlich zersplittertes und verteiltes System von Diasporas, die durch den einheitsstiftenden Rückbezug auf ein 'gelobtes Land، oder auf eine transnationale Organisation zusammengehalten werden. Vielmehr sind diese Transnationalen Sozialen Räume ein hybrides Produkt aus identifikativen und sozialstrukturellen Elementen der Herkunfts- und der Ankunftsregion, zwischen denen sie sich plurilokal und dauerhaft aufspannen. (Pries 2000: 416)

Wie bereits James Joyce (vgl. 2000: 118) distanziert sich Anil Bhatti (2015: 130) »von den trennenden Merkmalen der Homogenisierung (Rasse, Ethnie, Sprache und dergleichen mehr) « und vertritt ebenso wie Ludwig Wittgenstein die Ansicht, dass sich plurikulturelle und heterogene Gesellschaften als »ein kompliziertes Netz von Ähnlichkeiten, die einander übergreifen und kreuzen« (Witt-

4 | Vgl. die Fallstudie Salama 2016, insbes. das Fazit mit dem Titel: "Tristan - Tantris Transit", S. 18-20. 
genstein 1975: 57) gestalten. In Anlehnung an Samir Amin (vgl. 1999) folgert Bhatti:

Ähnlichkeit (similarity) wäre somit als universalistische, humanistische Perspektive zu verstehen. Sie beruht auf Solidarität, welche partikularistische Bindungen relativiert und an deren Stelle eine plurikulturelle Kommunikationsgesellschaft entwirft. Im Recht auf Ähnlichkeit und Solidarität könnte man vielleicht die Signatur von plurikulturellen Lebensformen sehen. (Bhatti 2015: 130, mit Verweis auf Amin 1999: 91)

Ebenso kann die Epistemik des Wissens als eine der Subkulturen dienen, an der eine Schnittmenge von Individuen bzw. Kollektiven teilhat. So gelten beispielsweise Kenntnisse über Mirabilien des Orients als enzyklopädisches, kosmographisches Wissen, das das Orientbild sowohl in orientalischer als auch europäischer Literatur konstituiert (vgl. u.a. Sivri 2016; Quenstedt 2017 u. 2018), sowie als Indiz für ein transkulturelles Wissen, das sich in jeder literarischen Form aufs Neue formt und neu schafft. ${ }^{5}$ Auf dieser Basis ergibt die Beschäftigung mit interkultureller Begegnung und Erkundung keine Etablierung von hegemonialen Dichotomien (Fremde /Andere und Eigene, Herkunft und Zielort usw.) und zielt auch nicht darauf ab, sämtliche Überlieferungswege zurückzuverfolgen, um einen vermeintlichen Anfangspunkt bzw. eine Urquelle zu fixieren. Sie dient vielmehr dazu, vor dem Hintergrund eines transkulturellen reziproken Prozesses die verschwungenen Linien nachzuzeichnen, um die Möglichkeiten und das Ausmaß der Erkundung zu analysieren, die Modi ihrer Zugehörigkeitsbereiche aufzudecken und die Verwobenheit der Kulturen offenzulegen. Für solch ein Verfahren stellt ein inter- bzw. transdisziplinärer Ansatz, der auch das Potential der Mehrsprachigkeit seiner Forscher ausschöpft, insbesondere bei der Beschäftigung mit philologischen Aspekten, eine sinnvolle Voraussetzung dar.

Hierfür ist sicherlich die institutionelle Re- und Neustrukturierung in Forschung und Lehre gefragt. Mit diesem Gedanken möchte ich für die stärkere Einbindung (vgl. ebenso Eibach/Opitz-Belakhal/Juneja 2012 und u.a. Heimböckel 2015) internationaler disziplinärer und interdisziplinärer Lehr- und Forschungsgebiete plädieren und vor allem die multilingualen Philologien dazu ermutigen, gemeinsam das »Projekt« der Interkulturalität (Heimböckel/Weinberg 2014; vgl. auch Bhatti 2015) zu intensivieren. Dieses sollte nicht das Ziel verfolgen, Gemeinsamkeiten und Differenzen hervorzuheben, sondern über die Analyse von Begegnungen und Modi der Erkundung, aber auch der Integrati-

5 | Dies aufzuzeigen, setzt sich der jüngst erschienene Band Literarische Orte in deutschsprachigen Erzählungen des Mittelalters zum Ziel, da die darin versammelten Beiträge, "die narrative Erzeugung literarischer Orte zu erfassen [suchen] und nach den besonderen Formen und Verfahren fragen, durch die Orte in mittelalterlichen Erzähltexten sprachlich hervorgebracht werden [...]. Die Art der Erschaffung von Orten in der erzählten Welt literarischer Texte stellt - neben der Tradierung und Funktionalisierung von Orten, den zweiten Schwerpunkt des Bandes dar." (Renz/Hanauska/Herweg 2018: 1) 
on von Subjekten, die Dynamik der Transkulturalität und die Mehrfachzugehörigkeit von Individuen (vgl. Welsch 2010) für ein empathisches Miteinander in Forschung und Lehre fruchtbar zu machen.

Mit diesem Plädoyer möchte ich nun die Aspekte von Interkulturalität kurz umreißen, die sich als mögliche Lehr- und Forschungsfelder einer Interkulturellen Mediävistik im Rahmen einer internationalen (also vor allem mehrsprachigen und disziplinübergreifenden $)^{6}$ Ausrichtung des Faches anbieten. Grundlage hierfür bildet meine Studie (die voraussichtl. 2018 erscheint), die die Forschungslandschaft und die Lehrinhalte der Mediävistik in Ägypten, speziell an der Germanistikabteilung der Philosophischen Fakultät der Universität Kairo, skizziert. Die bemerkenswerte Stellung komparatistischer Studien und jener Themen, die besondere geographische Räume als Kontaktzonen, historische Persönlichkeiten oder Begebenheiten, aber auch transkulturelles Wissen, markieren, wird darin angesprochen.

\section{Systematisierung einer Interkulturellen Mediävistik}

Im Folgenden geht es nun um den Versuch, Lehr- und Forschungsziele einer Interkulturellen Mediävistik zur Etablierung eines akademischen Lehrmoduls und Forschungsbereichs zu skizzieren, der sich an Heinz Sieburgs »Versuch einer Systematisierung eines Arbeitsfeldes« orientiert, »das zwar unter dem Begriff der Interkulturalität firmiert, dessen programmatische und methodische Spannweite aber keineswegs gegen das Konzept der Transkulturalität steht, sondern [...] dieses mit umschließt« (Sieburg 2015/2016: 399).

Angesichts der speziellen Situation der Mehrsprachigkeit in der Auslandsgermanistik kann sich das Forschungs- und Lehrgebiet einer interkulturell ausgerichteten internationalen Mediävistik als besonders ergiebig erweisen, da an ihm neue Verhandlungsräume entwickelt werden können. Innerhalb beider Fremdartigkeiten, einer interkulturellen Fremde innerhalb der polyvalenten Studentenschaft einerseits und des historisch Fremden der germanistischen Mediävistik andererseits, kann eine Kombination erwirkt werden, die zu einer gewinnbringenden anderen, weil neuen Vermittelbarkeit führt. Dementsprechend sind vor allem komparatistische Zugangsweisen besonders stark zu vertreten, da sich aus ihnen mehrere Arbeitsfelder ergeben und neue Horizonte eröffnen können. Als Grundlage für die Konzeptualisierung des Arbeitsfeldes und für den Entwurf des Moduls Interkulturelle Mediävistik dient, wie bereits oben erwähnt, ein für das Jahrbuch für internationale Germanistik erstellter Erfahrungsund Forschungsbericht über die akademische Lehre und Forschungslandschaft der ägyptischen Mediävistik an der germanistischen Abteilung der Philosophischen Fakultät der Universität Kairo (vgl. Salama 2018). Als wichtigste Erkenntnis aus der Einsicht in das Lehrangebot und in die Forschungsarbeiten ägypti-

6 | Dieter Heimböckel (vgl. 2015: 157-159) formuliert in seinem Vortrag Im Grenzgang sechs konkrete Maßnahmen für die Etablierung einer Germanistik als Schwellenkunde. 
scher mediävistischer Studien ist der komparatistische Zugriff zu beobachten und die Fokussierung auf Momente historischer Annäherung.?

Die folgende Tabelle dient der Veranschaulichung des erstellten literarischen Quellenparadigmas als »Projekt« (Heimböckel/Weinberg 2014) von interkulturellen Themen, Stoffen, Perspektiven, Motiven, aber auch von Texten mit inter- und transkulturellem Potential (unter besonderem Einbezug des mittelalterlichen arabischen Kulturraums). Diese Vorstellung basiert auf theoretischen und methodologischen Reflexionen, auf die zu Beginn in den Vorüberlegungen dieses Beitrags eingegangen worden ist und die bei der Systematisierung und Konzeptualisierung des Arbeitsfeldes der Kategorie Interkulturelle Mediävistik in Forschung und Lehre stets mitzudenken sind: $\mathrm{Zu}$ diesen zählen vor allem vergleichende Prozesse der Adaption und ihrer Reziprozität. Darin sind ethnographische, topographische, imagologische Berührungen, Kontakt- und Konfliktpotentiale zwischen Kulturen und Kulturkreisen, als Grundlage für Vorbildhaftigkeit, Identitätskonstruktion, Migration und Austauschprozesse, mit inbegriffen. ${ }^{8}$ Es geht also dabei um die Präzisierung einer komparatistisch angelegten Untersuchungskategorie, die als Tertium Comparationis zwischen den verschiedensprachlichen Texten und Kulturen, wobei Kultur - verstanden als Text - als Textur, also als Gewebe, das aus verschiedenen Fasern besteht, fokussiert wird. Da eine Durchdringung und Verwobenheit ihrer Fasern jeder Kultur inhärent ist, kann sie als transkulturell definiert werden. Geht man von einem fluiden Kulturbegriff aus - wie oben ausgeführt -, erscheint es bei einer farblichen Melange bzw. einer Durchdringung als äußerst schwierig, fast unmöglich, diese in ihre ursprünglichen Grundfarben vereinzelt zurückzuverfolgen. Ein Versuch, diese Melange in ihre einzelnen Pigmente aufzulösen, kann stets nur in eine unendliche Anzahl von Analysen münden. Das Aufzeigen von Ähnlichkeit (Bhatti), die auf transkulturelle Prozesse der Verflechtung zurückgeht,

7 | Als eine der herausragendsten Herausforderungen in Bezug auf außersprachliche v.a. historische Kenntnisse, stellt sich die unterschiedliche Zeitrechung mittelalterlich arabischer - vor und nach der Hidschra (entspricht etwa dem Jahr 622 nach Christus) - und europäischer Geschichtsbücher (vor und nach Christus) dar. Außerdem wird der dem europäischen Mittelalter adäquate Zeitraum der arabischen Welt als islamische Epoche bezeichnet, die meist mit den islamischen geistigen, wissenschaftlichen und kulturellen Errungenschaften assoziiert wird und in Kontrast zum dunklen Mittelalter Europas steht. Damit stellt sich die Frage, wie die arabische Geschichte, deren mittelalterliche Kultur und Dichtung, auch und vor allem zum näheren Verständnis der eigenen und der anderen bzw. fremdsprachlichen mittelalterlichen Kultur und Geschichte, mit in die akademischen Lehrpläne und Forschungsfelder einbezogen werden können.

8 | Norbert Mecklenburg (2008: 93f.) betrachtet das Erzählen als "transkulturelles Phänomen, da gewisse Erzähltechniken wie das personale Erzählen über kulturelle Grenzen hinaus verbreitet sind." "Interkulturelle Dimensionen“ des Erzählens begreift er als "kulturspezifische Strategien", "die in literarischen Übersetzungen oder Erzählungen zum Ausdruck kommen". 
ist darin ebenso Teil des interkulturellen Arbeitsbereichs wie das Aufspüren von Momenten der Differenz, von Prozessen der kritischen Reflexion, der Konfrontation, aber auch der Assimilation. Diesen Momenten wohnt »ein genuin grenzüberschreitendes Potenzial« inne, das Bedeutsamkeit konstituiert (Dembeck 2017: 14), wie beispielsweise über das Staunen (vgl. Heimböckel/Weinberg 2014), welche »von Kultur fortlaufend erzeugt« wird und sich also »potenziell ständig verändert« und »niemanden unhintergehbar auf etwas « festlegt (Dembeck 2017: 14; Hervorheb. i.O.).

Tabelle 1: Konzeptualisierung von Interkulturalität im Rahmen einer transkulturellen und transdisziplinären Mediävistik in Forschung und Lehre als Projekt

\begin{tabular}{|c|c|}
\hline Perspektive & Aspekt: Thematik/Quellen \\
\hline $\begin{array}{l}\text { I. Faktuales: } \\
\text { Historische } \\
\text { Schnittpunkte }\end{array}$ & $\begin{array}{l}\text { - historisch weitreichende Ereignisse (Kreuzzüge, } \\
\text { Franken und Araber) } \\
\text { - historische Persönlichkeiten mit transkulturellem } \\
\text { Profil als Grenzgänger der Kulturen (Karl der Große } \\
\text { und Harun ar-Raschid: Roger II.; Friedrich II. von } \\
\text { Staufen u.a.) } \\
\text { - Kontakt- und geographische Konfliktzonen } \\
\text { als Brenn- und Schnittpunkt der Kulturbegegnung: } \\
\text { Handels-, Pilger- und Kreuzfahrten } \\
\text { - Epistemik usw. }\end{array}$ \\
\hline $\begin{array}{l}\text { 2. Universalia: } \\
\text { Transkulturelle } \\
\text { Texte: enzyklo- } \\
\text { pädische und } \\
\text { anthropologi- } \\
\text { sche Texte/ als } \\
\text { gemeinsames } \\
\text { Kulturgut }\end{array}$ & $\begin{array}{l}\text { - Die Geschichten aus } 1001 \text { Nacht } \\
\text { - Secretum Secretorum } \\
\text { - Al Firdausi/Qazwini/Averroes Kommentare } \\
\text { zu Aristoteles } \\
\text { - Alexanderstoff - Alexanderroman } \\
\text { - die Mystik des Al Ghazali } \\
\text { - Nikomachische Ethik: Wertesysteme unterschiedlicher } \\
\text { Kulturen bzw. Kulturstufen samt ihrer spezifischen } \\
\text { Literaturausprägungen, die Konstruktion des Glücks: } \\
\text { Willens- und Entscheidungsfreiheit, Tugenden } \\
\text { und Ethik, Verstand und Lust } \\
\text { - Liebespoesie } \\
\text { - Sagen und Motivstoffe } \\
\text { - Kalila wa Dimna/Äsop } \\
\text { - transkulturelle Kartographie (die Ebstorfer Weltkarte } \\
\text { zitiert beispielsweise in ihrer Legende } \\
\text { Isidors Etymologiae; al-Idrisi) }\end{array}$ \\
\hline
\end{tabular}




\begin{tabular}{|c|c|}
\hline Pers & spekt: Thematik/Quellen \\
\hline $\begin{array}{l}\text { 3. Fiktives: Kultur- } \\
\text { poetik: Motiv- } \\
\text { ähnlichkeiten: } \\
\text { volkssprachliche } \\
\text { mittelalterliche } \\
\text { literarische Texte } \\
\text { und strukturelle } \\
\text { Ähnlichkeiten } \\
\text { der Narrative } \\
\text { auch bei unkla- } \\
\text { ren Überliefe- } \\
\text { rungswegen }{ }^{10}\end{array}$ & $\begin{array}{l}\text { - orientalische Figuren (Weiblichkeits-, Männlichkeits- } \\
\text { konstrukte) mit »orientalisierender« Kulisse } \\
\text { - Reisetexte: Mirabilien - ágāib als Teil des Wissens: } \\
\text { Enzykolpädisches und Wunderbares (Megenberg; } \\
\text { vgl. Bein 2010; Quenstedt 2017; 2018) } \\
\text { - hybride Gestalten: Feirefiz, Cundrie, Arabel } \\
\text { - Wundervölker (imagologische Aspekte - Konstruktion } \\
\text { von Stereotypen) } \\
\text { - Macht- und Herrschaftsstrukturen als hegemoniale } \\
\text { und/oder kolonialisierende Konstrukte usw. }\end{array}$ \\
\hline $\begin{array}{l}\text { wissen- } \\
\text { liche } \\
\text { n }\end{array}$ & $\begin{array}{l}\text { - Heldenbilder und Hybridität als interkulturelle } \\
\text { Konstellation (Heldendichtung: die Konstruktion } \\
\text { eines mythischen Helden [Ikone]; Schicksalhaftigkeit } \\
\text { und Handlungsautonomie/Heldenethos) } \\
\text { - die kollektive Verarbeitung von Traumata } \\
\text { und memoria: (Ethos; Ehre und Treue) } \\
\text { - Körper, Macht, Raum, Zeit, Erinnerung } \\
\text { und Gedächtnis, Emotionen und Affekte, } \\
\text { Multilingualität und Sprachdifferenz usw. }\end{array}$ \\
\hline
\end{tabular}

\section{HERZOG ERNST (B) UND Die Geschichten AUS 1001 NACHT}

Wie kein anderes Werk dienten Die Geschichten aus 1001 Nacht zahlreichen Werken als Fundus orientalischer Stoffe und Motive. An dieser Stelle soll der Hinweis darauf, dass sich Die Geschichten aus 1001 Nacht selbst aus verschiedenen Quellen zusammensetzen, genügen, um diesen Text als transkulturell zu markieren (vgl. u.a. Sivri 2016: 114-174). Die Darstellung zahlreicher Parallelen zwischen einigen Geschichten der Geschichtensammlung 1001 Nacht und dem mittelalterlichen Versepos Herzog Ernst (B) (im Folgenden HE [B]) soll veranschaulichen, welches Potential beide Texte sowohl für das Studium als auch für die Erforschung von Transkulturalität im Rahmen einer interkulturellen Medi-

9 | Ebenfalls unklar ist und bleibt, wer wen zuerst beeinflusst hat. Dennoch muss der Dialog offen bleiben. Die Aufgabe einer Interkulturalität in der Mediävistik sollte darin bestehen, der Konstruktion von Feindbildern durch Offenheit und Annäherung entgegenzuwirken. 
ävistik bergen..$^{\circ}$ Die in der Forschung erwiesenen Parallelen verweisen auf kulturelle bzw. auf transkulturelle Schnittpunkte und Narrative, deren verflochtene Überlieferungswege zwar nicht definitiv nachgezeichnet, jedoch stets vor dem Hintergrund eines verflochtenen Texturbegriffs von Wissen mitgedacht werden sollten. Als Tertium Comparationis zählt das >Wunderbare $<-$ als mirabilia (arab. ágāib) -, das zwischen Faktizität und Fiktionalität oszilliert, zum enzyklopädischen Wissen, das das Orientbild - selbst im Orient - konstruiert. ${ }^{11}$ Die Untersuchung des Orientbildes anhand einer detaillierten Analyse des Reiseteils des $H E$ (B) sollte nicht als Selbstzweck erfolgen, sondern stets mit der Frage nach dessen Funktionalisierung im jeweiligen Werk verknüpft werden. ${ }^{12}$ Um es nicht nur bei einem deskriptiven Ansatz zu belassen, der zweifellos zu den ersten Arbeitsschritten gehört, sollte des Weiteren ein Analyseverfahren gewählt werden, das nicht allein das komparatistische Fixieren von motivischen und narrativen Analogien oder Unterschieden verfolgt, sondern darüber hinaus die Frage nach dem Fremdsein, seinen Erscheinungsformen und seiner Wirkung auf figuraler Ebene stellt, als dessen Auswirkung Momente des Staunens, des Wunderns verstanden werden.

\subsection{Analogien der Motive und Narrative zwischen HE (B) und den Geschichten aus 1001 Nacht $^{13}$}

Im ersten Arbeitsschritt wird das Ziel verfolgt, mittels eingehender Lektüre der drei Geschichten aus 1001 Nacht, der Geschichte von Sindbad dem Seefahrer (sieben Reisen), ${ }^{14}$ der Geschichte des dritten Bettelmönches ${ }^{15}$ und der Geschichte des

10 | Hinweise auf weitere mittelalterliche trans- und interkulturelle Quellentexte, die auf Kulturkontakte zwischen europäischen und arabischen Vertretern verweisen, diese thematisieren bzw. den Austausch implizieren, finden sich in Tab. 1.

11 | Vgl. Salama (2003), darin auch ausführlich zu motivischen Parallelen zwischen dem HE (B) und den Geschichten aus 1001 Nacht: die Erzählungen von Sindbad dem Seefahrer, Die Geschichte des dritten Bettelmönchs und Die Geschichte des Prinzen von Karisme und der Prinzessin von Georgien, S. 194-236. An dieser Stelle sei auf das vielversprechende laufende Dissertationsprojekt von Falk Quenstedt verwiesen: Das exotische Wunderbare zwischen Literatur und Wissen in mittelhochdeutschen und arabischen Texten des 10. bis 13. Jahrhunderts: Einsicht in die Projektbeschreibung bietet die SFB-Website; online unter: http://www.sfb-episteme.de/teilprojekte/zeigen/ B02/up_quenstedt/index.html [Stand: 1.9.2018].

12 Siehe hierzu u.a. folgende Arbeiten: Szklenar (1966), Salama (2003), Sivri (2016).

13 Im Folgenden werden die Ergebnisse meiner Arbeit zum $H E(B)$ zusammengefasst. 14 | Zitiert wird aus der Ausgabe Enno Littmanns (vgl. Die Geschichte von Sindbad dem Seefahrer 1953), angegeben mit der Sigle "Li IV" und Seitenzahl.

15 | Zitiert wird aus der Ausgabe Enno Littmanns (vgl. Die Geschichte des dritten Bettelmönches 1953), angegeben mit der Sigle "Li l« und Seitenzahl. 
Prinzen von Karisme und der Prinzessin von Georgien, ${ }^{16}$ Parallelen in Motivik und strukturellen Narrativen zum Reiseteil des $H E(B)^{17} \mathrm{zu}$ markieren. Die folgende Tabelle wurde auf der Basis der größtenteils aus meiner Dissertation von 2003 zusammengetragenen Parallelen erstellt und hier zur besseren Überschaubarkeit nach den jeweiligen motivischen und narrativen Strukturen des HE (B) angeordnet und skizziert, um als Grundlage für die darauffolgende komparatistische Herausarbeitung von Momenten des Staunens zu dienen (vgl. Tab. 2).

Die synoptische Darlegung von Parallelen zeigt deutlich, dass die Konstruktion eines fabulösen und wunderbaren Orients bereits in den orientalischen Sammlungen vorliegt und über den Transfer beibehalten bzw. verändert und/oder evtl. - wie im HE (B) - um weitere Elemente erweitert worden ist (vgl. Salama 2003: 194-236). Die michel wunder (HE [B]: v. 2), die das Wunderbare als enzyklopädisches Wissen in die Konstruktion des Orientbildes mit einschließen, fungieren somit nicht (allein) als »attractive sugarcoating « (Spuler 1983: 410), sondern sind Teil eines transkulturellen Wissens und einer Wissensvermittlung über den Orient, an dem sowohl Orient wie Okzident teilhaben. ${ }^{18}$

\subsection{Momente des Staunens zwischen HE (B) und den Geschichten aus 1001 Nacht als inter- und transkulturelle Untersuchungskategorie}

Als weitere Untersuchungskategorie werden nun Momente des »Staunens« herangezogen, die Dieter Heimböckel und Manfred Weinberg als »liminales Phänomen«, »beständig wie auf einer Grenze«, deuten, das »den Beobachter aus den Fesseln seiner Kulturbedingtheit« löst (Heimböckel/Weinberg 2014: 121). Die Autoren Heimböckel und Weinberg verweisen auf Renate Schlesier, wenn sie im Staunen

16 | Zitiert wird aus der Ausgabe Martin Schillers (vgl. Die Geschichte des Prinzen von Karisme 1926), angegeben mit der Sigle "Sch" und Seitenzahl.

17 | Zitiert wird aus: Herzog Ernst. Ein mittelalterliches Abenteuerbuch (1972).

18 | Jan Dirk Müller versteht "die Begriffe ,enzyklopädisches Erzählen und renzyklopädischer Romanı "nicht als Struktur-, sondern als Funktionsbezeichnungen [...]: Kriterium sei die - bisweilen programmatische - Belehrung über Wissbares, mithin die wissensvermittelnde Funktion der Texte in enzyklopädischer Absicht und unter je spezifischen medialen Bedingungen. Zu diesem Zweck böten sich insbesondere die narrativen Strukturschemata der Reise, der Ausbildungsbiographie und des historischen Romans an." (Noll 2015) 


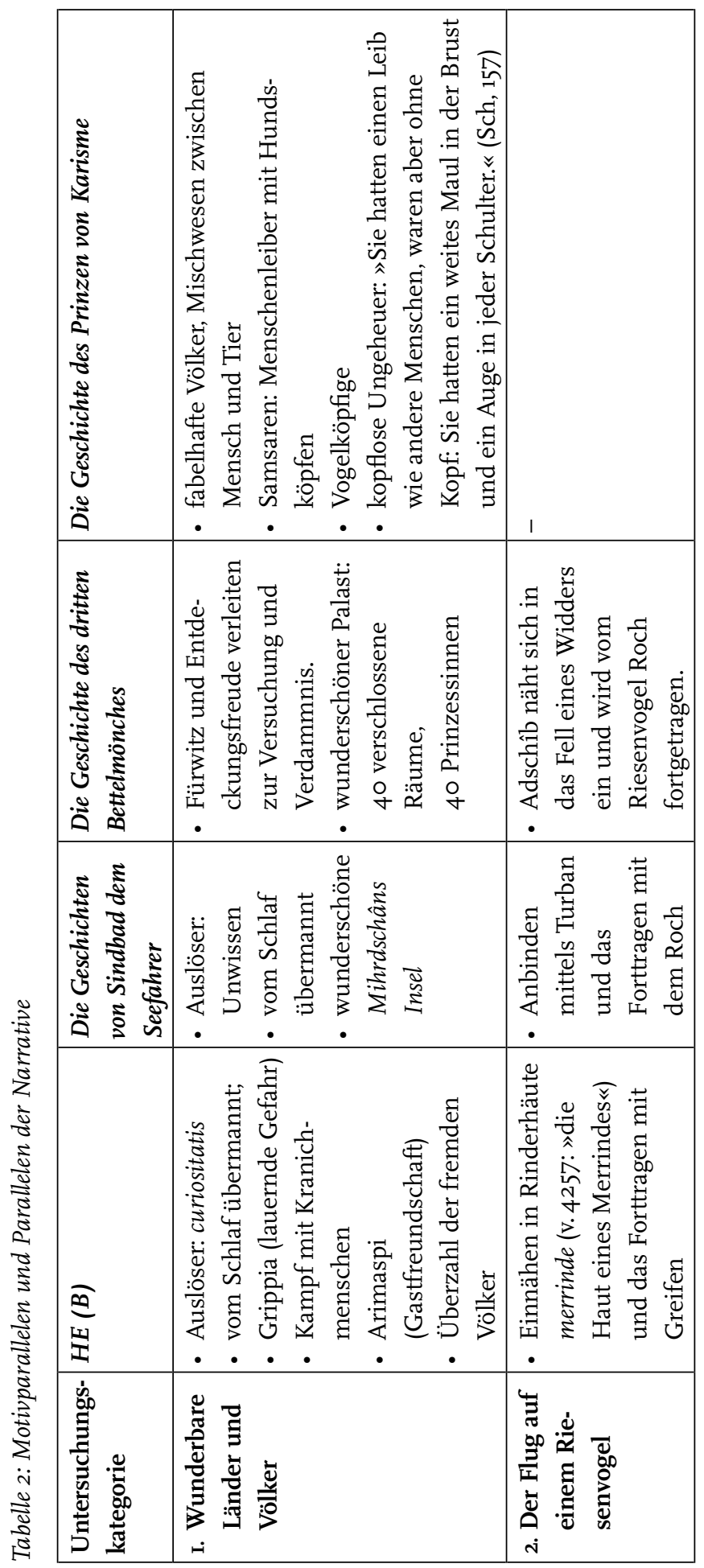




\begin{tabular}{|c|c|c|c|}
\hline 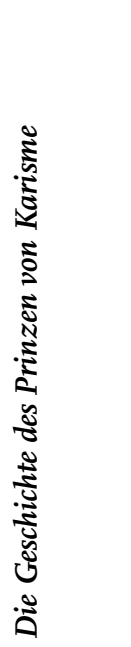 & 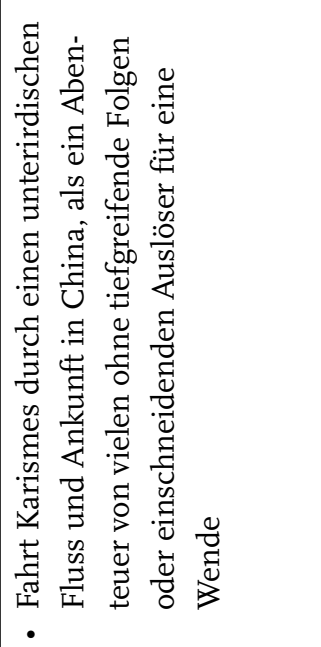 & 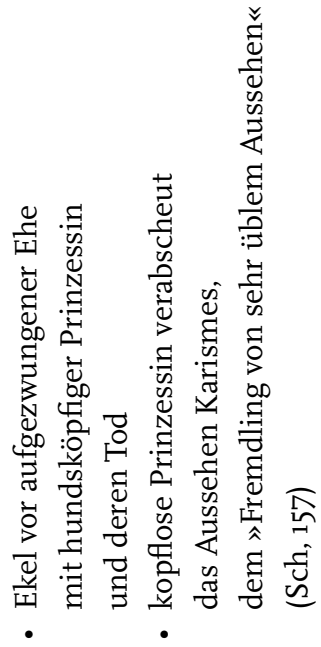 & 1 \\
\hline 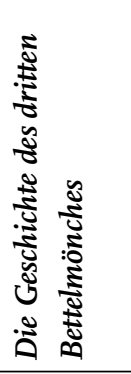 & 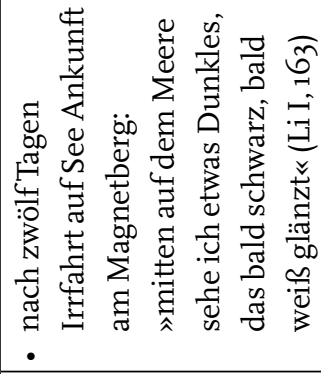 & 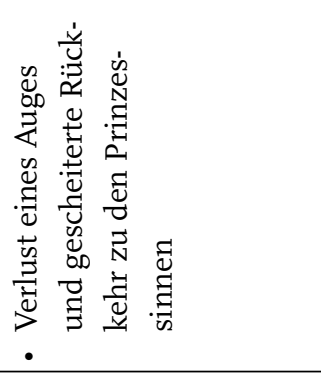 & \\
\hline 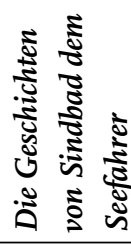 & 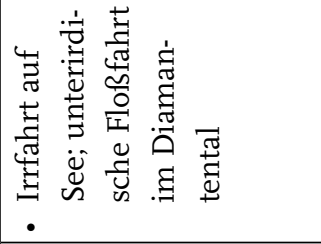 & 1 & 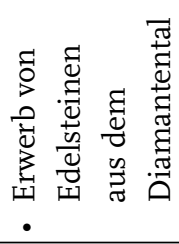 \\
\hline 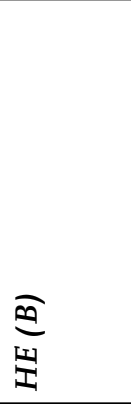 & 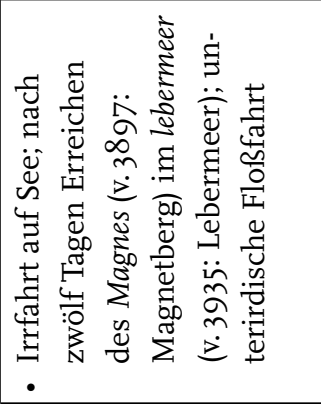 & 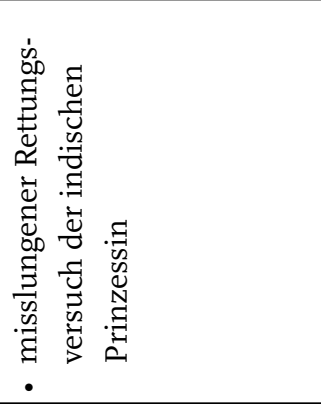 & 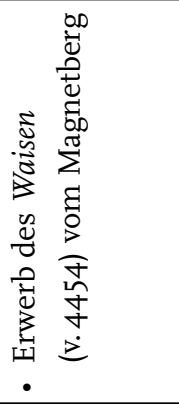 \\
\hline 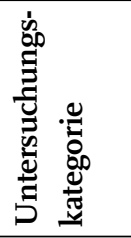 & 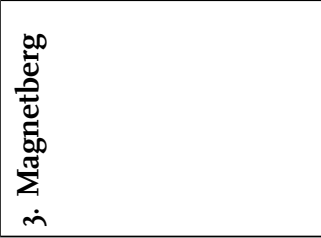 & 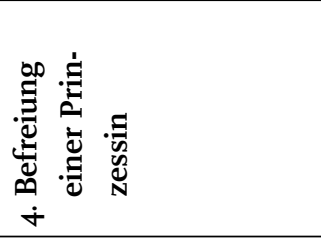 & 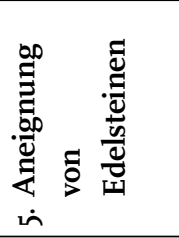 \\
\hline
\end{tabular}




\begin{tabular}{|c|c|c|c|}
\hline 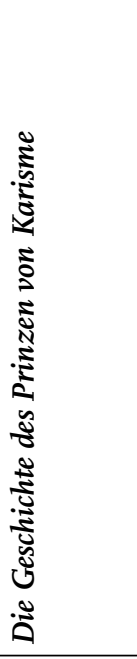 & 1 & 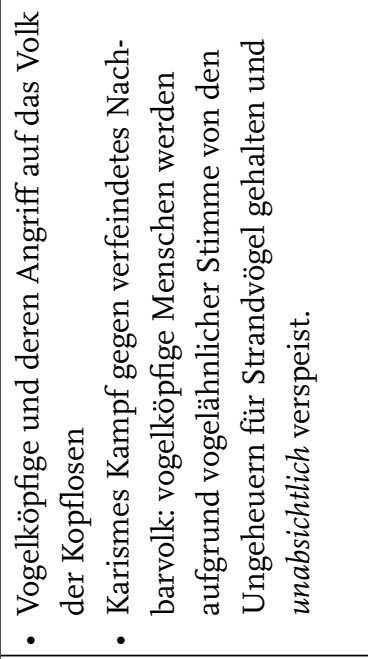 & 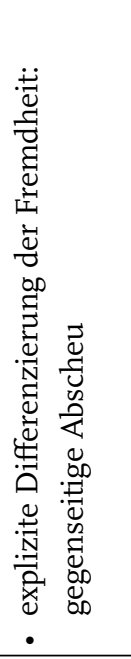 \\
\hline 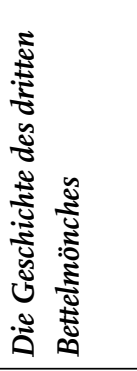 & 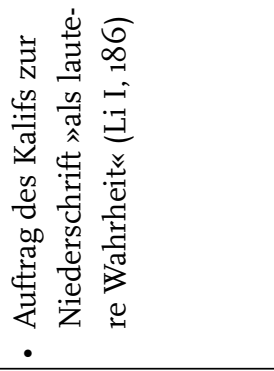 & 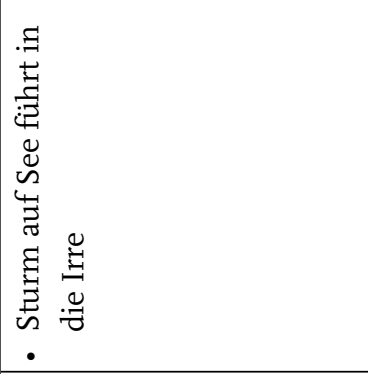 & 1 \\
\hline 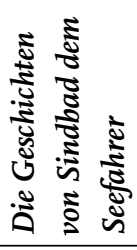 & 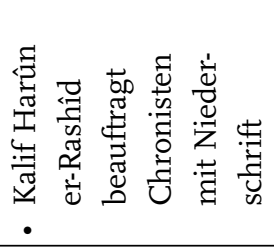 & 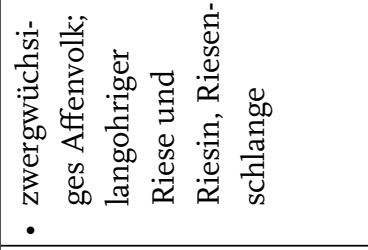 & 1 \\
\hline 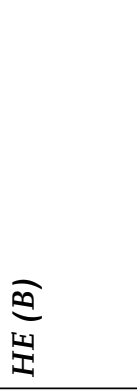 & 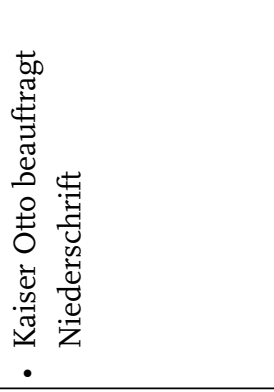 & 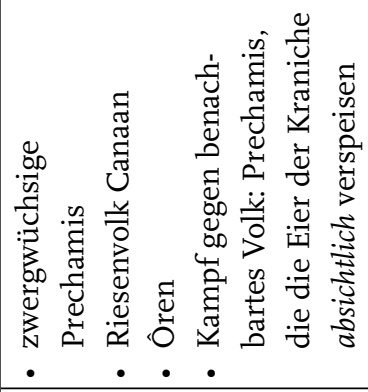 & \\
\hline 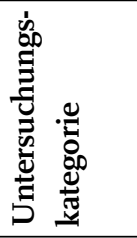 & 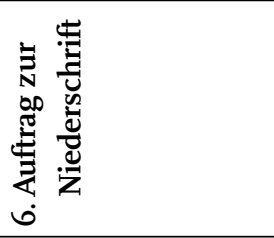 & 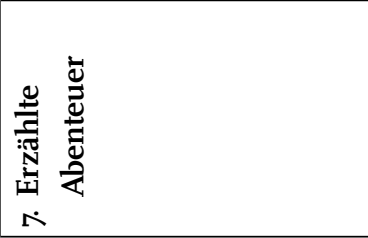 & 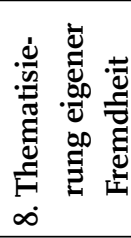 \\
\hline
\end{tabular}


einen auf absolute Wertmaßstäbe verzichtenden Kulturvergleich [erkennen], indem es nicht nur "die Selbstverständlichkeit eigener kultureller Erfahrungen durchbricht" (Schlesier 1996: 155), sondern auch interkulturelle Grundlagenkategorien wie das Eigene und das Fremde selbst in Frage stellt. Insofern übernimmt das Staunen in der interkulturellen Konstellation gewissermaßen eine doppelte Funktion: und zwar die des Vehikels ebenso wie die der Irritation. (Heimböckel/ Weinberg 2014: 121)

In der »perspektivischen Offenheit des Projekts«, wie Heimböckel/Weinberg es vorschlagen, werde es möglich, »Interkulturalität, im Kontext von Grenzen einerseits und Nichtwissen und Staunen andererseits zu reflektieren.« (Ebd.) In ihren weiteren Ausführungen vertreten sie die Ansicht, dass erst der »liminale Raum [...] [als] der eigentliche Ort nicht des Wissens, sondern des Nichtwissens als Gegenstand der Interkulturalität« (ebd.: 131) fungiert.

Im folgenden Arbeitsschritt versuche ich, Momente des Staunens aber auch des Wunderns als Tertium Comparationis in beiden Texten als Übergang von Nichtwissen in Wissen zu markieren. Den Ausführungen Heimböckels und Weinbergs zufolge beschreibe ein solches Staunen

keinen Mangel, sondern einen Gewinn. Und der Gewinn liegt im Bewusstwerden eines Nichtwissens, das das angeeignete bzw. gelernte Wissen überrascht. [...] Nichtwissen ist in diesem Fall nicht die Kehr- oder dunkle Seite des Wissens, sondern ein Korrektiv zu der im Wissen angelegten Strategie der Vereinnahmung dessen, was gewusst werden soll, und zugleich der Modus, der auf Anerkennung des Nichtgewussten zielt. (Ebd.: 134)

Man kann in diesen Momenten des Transfers ein Wissen erkennen, dessen Konturen als transkulturelles Wissen vom und über den Orient in beide Texte einfließt und diesen als einen »vom Horizont eingeräumten Raum « (ebd.: 140) konzipiert. Der von Heimböckel/Weinberg in Anlehnung an Udo Schöning (vgl. 2000) vorgeschlagene Begriff des Horizonts als »angemessenes Raumkonzept des Projekts der Interkulturalität« impliziert einen offenen Wissenshorizont, in dem »die Anerkenntnis des Nichtwissens, das sich nicht im Bezug auf fixe Grenzen etablieren kann« (Heimböckel/Weinberg: 140), als Dimension integriert ist.

So erscheint der Horizont als jenes, das dem oben als liminales Phänomen، benannten Staunen erst einen Raum gibt, in dem es statthat. Das Staunen und die von ihm immer wieder initiierte Verschiebung beziehen sich zwar auf Grenzen, aber auf notwendig verflüssigte. Der Horizont zeigt sich so auch als angemessenes Raumkonzept des Projekts [Hervorheb. i.0.] der Interkulturalität, das auf einer solchen Verschiebung 'beruht, die als Prozess nicht nur die räumliche Verschiebung von Grenzen meint (die im Bereich des Gewussten verbliebe), sondern auch die Anerkenntnis des Nichtwissens, das sich nicht im Bezug auf fixe Grenzen etablieren kann, sondern wiederum nur im vom Horizont eingeräumten Raum. (Ebd.: 140) 
Im Folgenden soll die Erstellung einer Tabelle (vgl. Tab. 3) dazu verhelfen, einen Einblick in Modi des Staunens aus beiden Texten zu vermitteln. Hierin werden beispielhaft explizite Momente des Staunens aufgenommen, dessen Auslöser sich dem Verstehen und der Erklärung entziehen (vgl. Tugendhat 2004: 159), und jeweils nach dem kontextuellen Anlass des Staunens oder des Wunderns kategorisiert. Zum Schluss werden die daraus erschlossenen Erkenntnisse zur Beantwortung der Frage nach ihrer jeweiligen Funktionalisierung zusammengetragen. Damit wird sowohl die Offenheit der Grenze als auch deren Liminalität, in der feindliche bzw. friedliche Aushandlungsmöglichkeiten vorgestellt werden, sichtbar. An diesen Affekten, die teils von ähnlichen, teils von unterschiedlichen Auslösern in Gang gesetzt werden, haben beide Subjekte bzw. Kulturen teil. Schließlich kann sich die aus der interkulturellen Begegnung und Erkundung entstandene subjektgebundene Reaktion auf beiden Seiten über verschiedene Modi verbalen wie auch nonverbalen Handelns ausdrücken und jeweils ähnliche bzw. differenzierte Facetten annehmen.

\section{Conclusio}

Die Beschäftigung mit beiden Texten hat nicht nur Analogien in den Motiven und den Narrativen in Bezug auf das Wunderbare und Phantastische ergeben, sondern es konnten auch Analogien in den Anlässen für das Staunen und das Wundern festgestellt werden. Auf der Ebene der Narration fungiert das Staunen meist als retardierendes und irritierendes Moment, häufig aber auch als hyperbolisches Mittel zur Hervorhebung von Superlativen, wie beispielsweise besonderen übernatürlichen Leistungen, so z.B. der Allmacht Gottes, der Klugheit und Tapferkeit des Protagonisten und seiner Heldentaten, die durch die Exklusivität der errungenen Kuriosa und Wunderwesen bisher unentdeckter Völker und Länder hervorgehoben werden. Es lässt sich feststellen, dass in beiden Texten das Staunen über die erzählten Abenteuer den Anstoß bzw. die Rechtfertigung zu deren Verschriftlichung geben. Mit der Überführung von einem mündlich tradierten Erlebnis in die eigenen Bücher erfolgt sowohl eine interkulturelle wie auch intermediale Wissensübertragung und -aneignung, die den Wissenshorizont und das Erzählgut des jeweiligen Reiches (Harun ar-Raschid bzw. Kaiser Otto) um neue Erlebnisse und Wunder des Orients erweitert. Visuell versinnbildlicht wird dieser Wissenserwerb beispielsweise im HE (B) mit der Eroberung des »Waisen « und seiner Fixierung in die Kaiserkrone und in den Geschichten Sindbads über die erworbenen Exotika und die gewonnenen Schätze. Der königliche Beschluss dient jeweils dem Ruhm des eigenen exklusiven Königreichs, das sich staunenswerte Erlebnisse zu eigen macht, indem es sie in seinen Erzählfundus aufnimmt. Das Wissen vom Anderen, vor allem auch den Sitten und Sprachen fremder Völker, erweitert in beiden Texten das Reich nicht nur um Kuriosa und kostbare Kulturgüter, sondern auch und vor allem um geistige Schätze, wie kognitive Fähigkeiten, die sich über interkulturelle Begegnungen und Erkundungen fremder Orte zu gewinnbringenden Erkenntnissen ausbilden. 


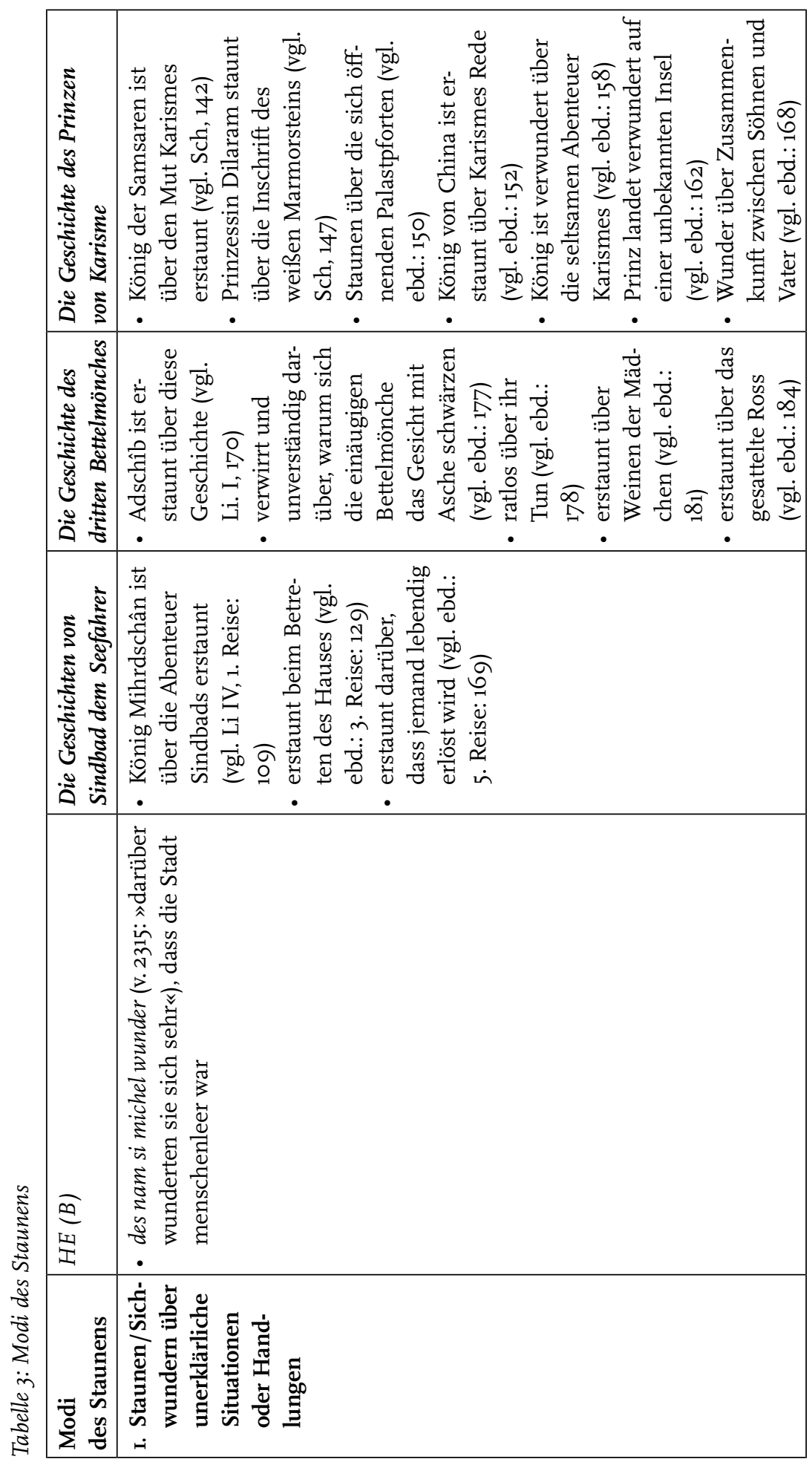




\begin{tabular}{|c|c|c|}
\hline 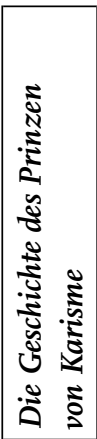 & 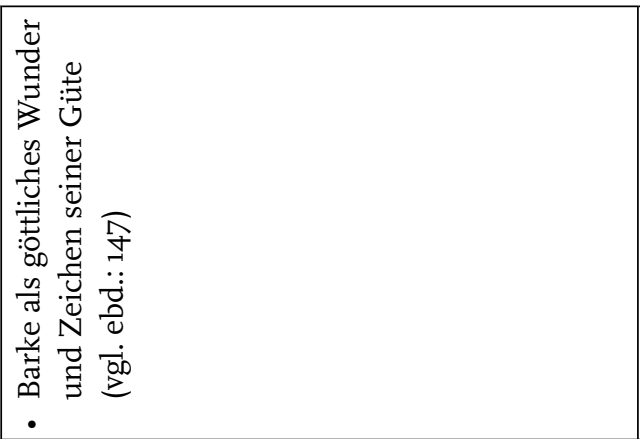 & 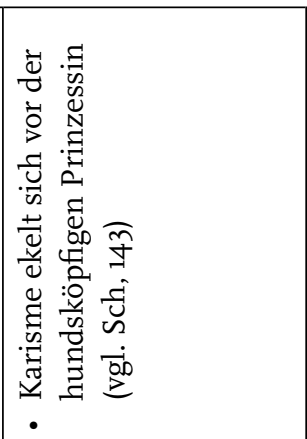 \\
\hline 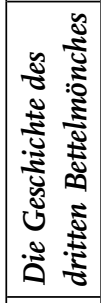 & & \\
\hline 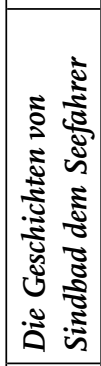 & 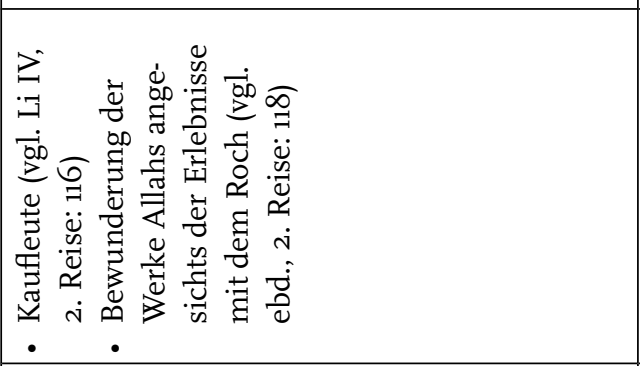 & 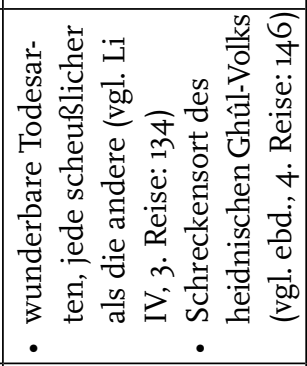 \\
\hline 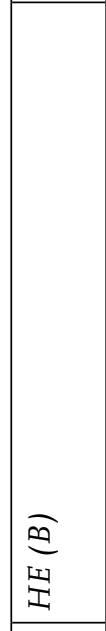 & 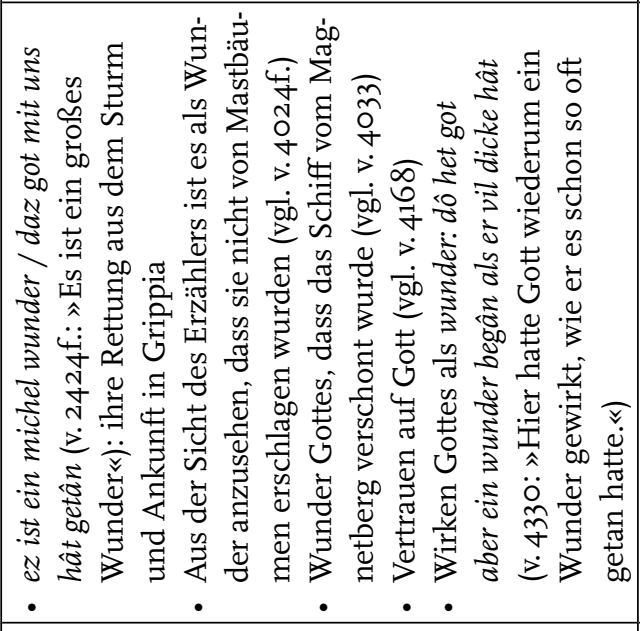 & 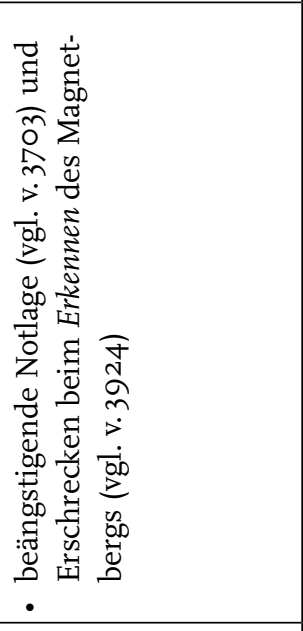 \\
\hline 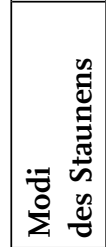 & 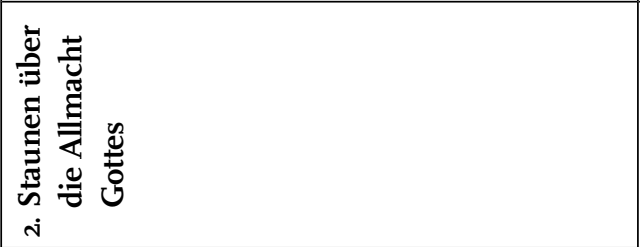 & 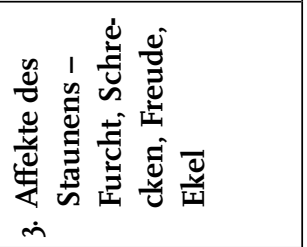 \\
\hline
\end{tabular}




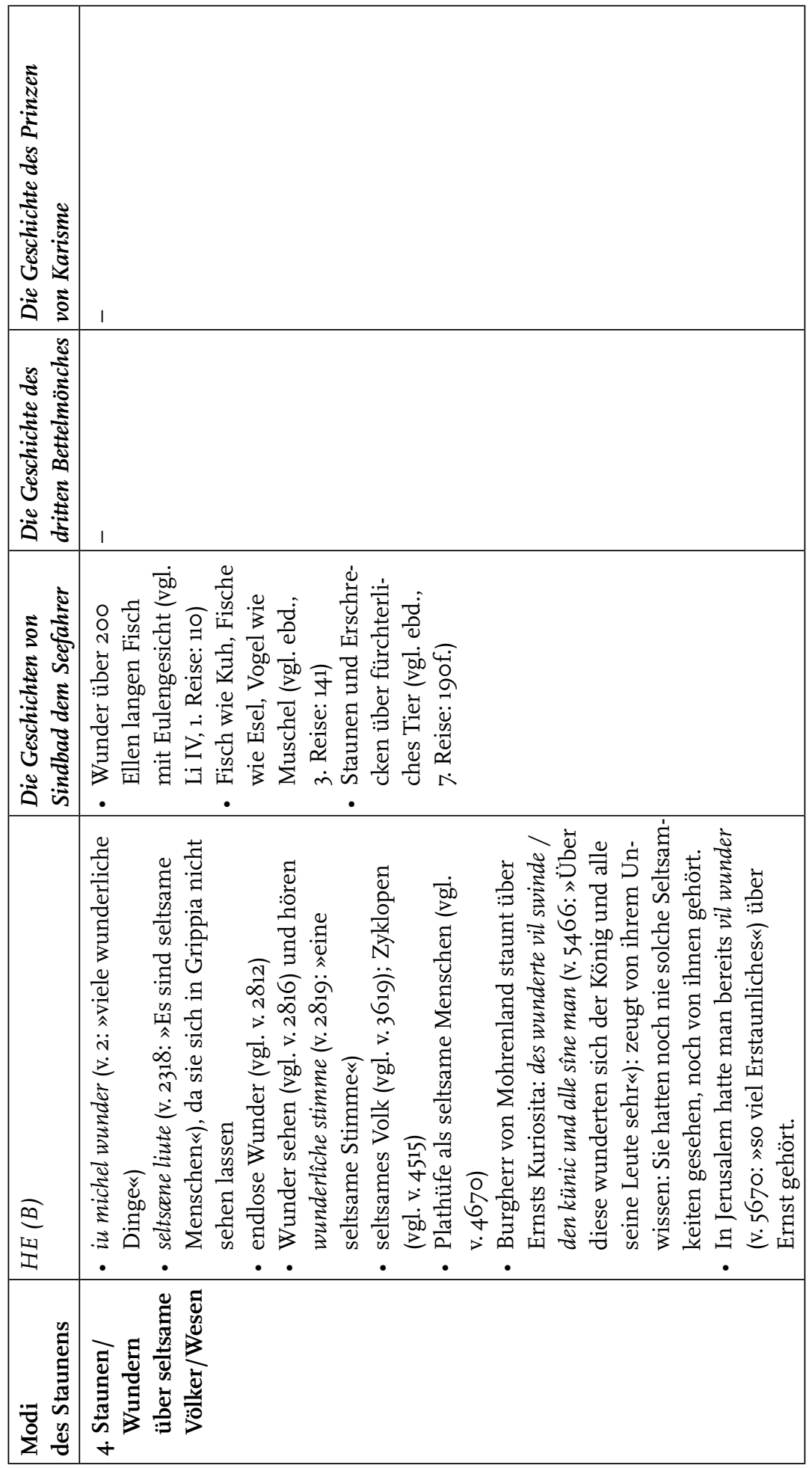




\begin{tabular}{|c|c|c|}
\hline 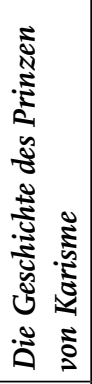 & 1 & 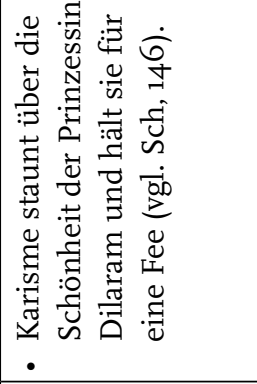 \\
\hline 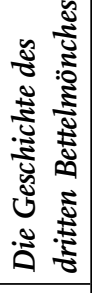 & \begin{tabular}{|l} 
\\
\end{tabular} & 1 \\
\hline 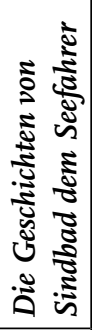 & 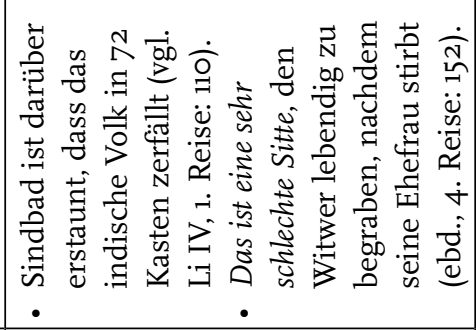 & \\
\hline$\frac{\infty}{2}$ & 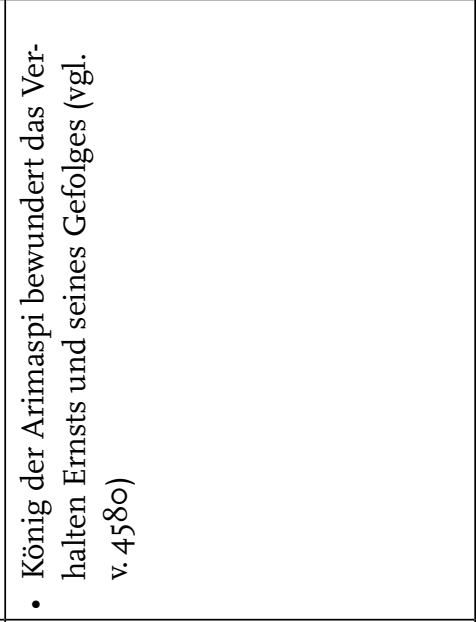 & 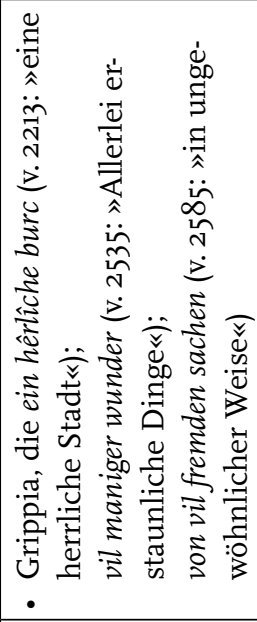 \\
\hline 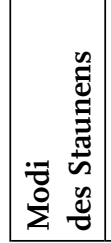 & 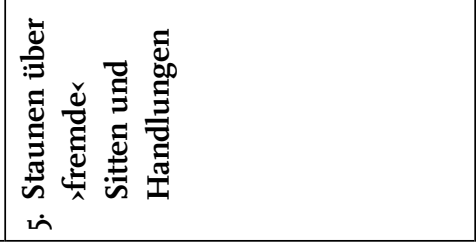 & 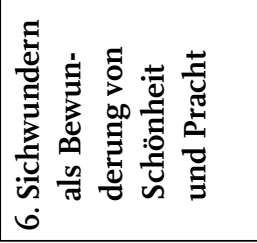 \\
\hline
\end{tabular}




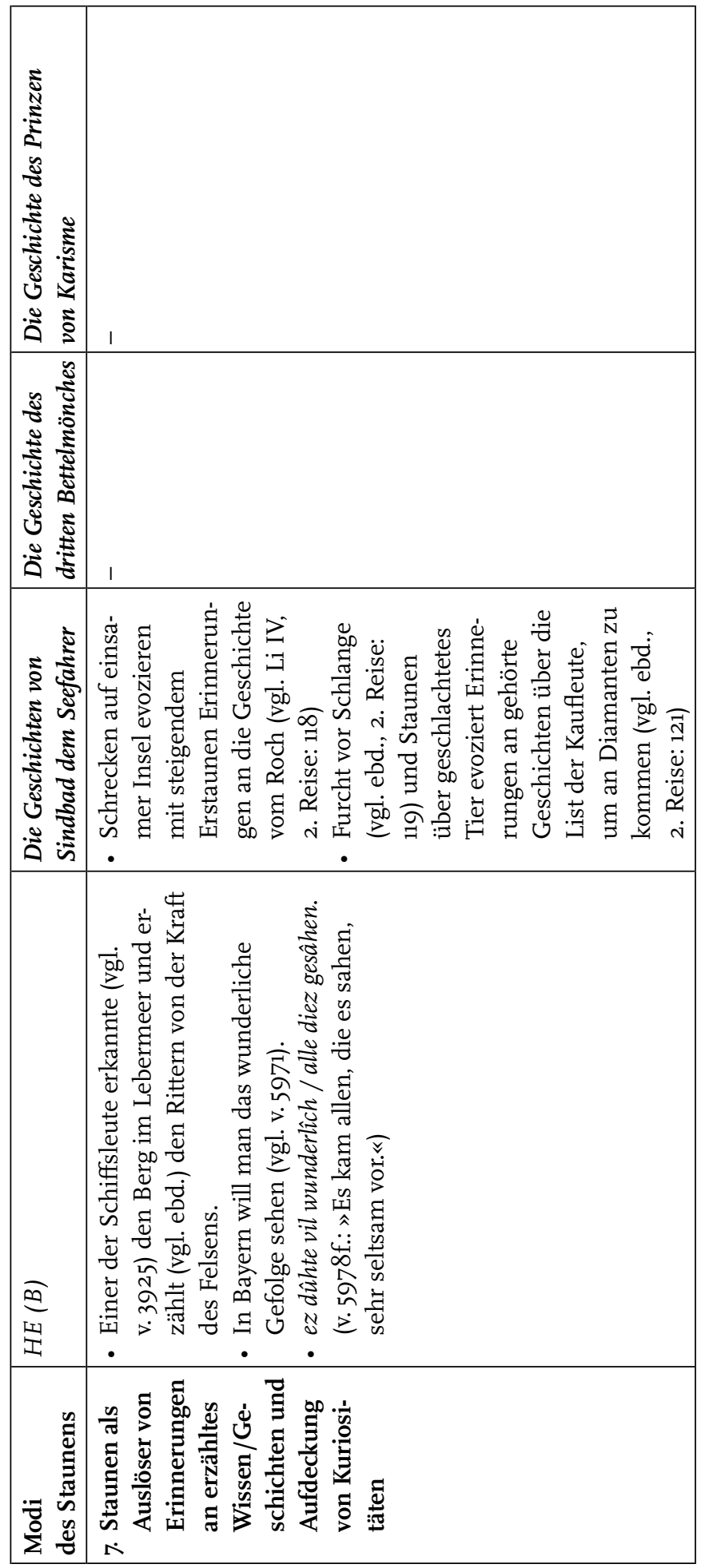




\begin{tabular}{|c|c|c|}
\hline 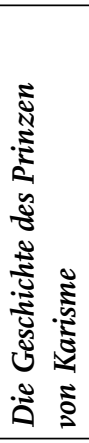 & 1 & 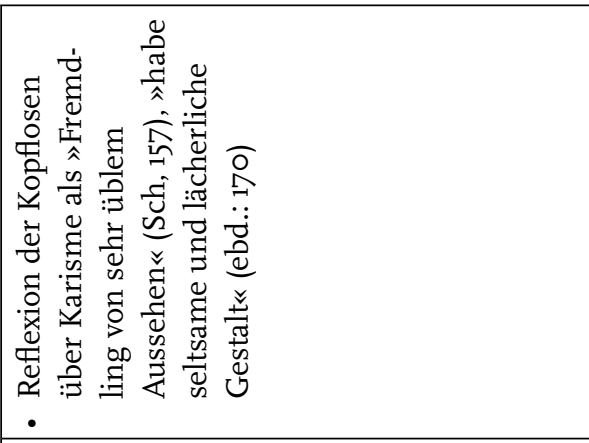 \\
\hline 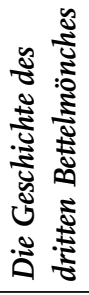 & 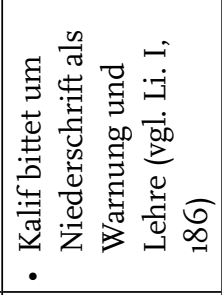 & 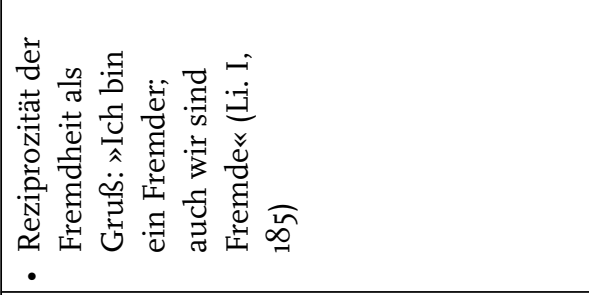 \\
\hline 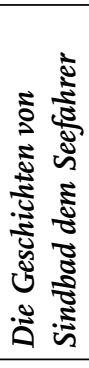 & 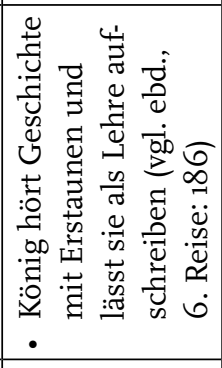 & 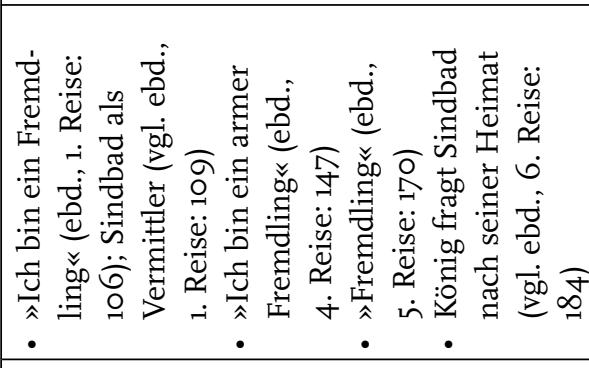 \\
\hline sa & 1 & 1 \\
\hline 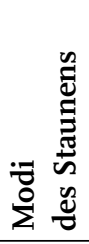 & 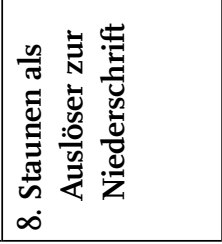 & 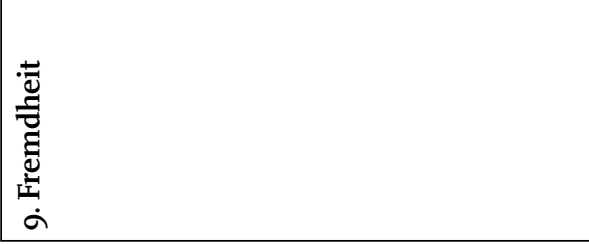 \\
\hline
\end{tabular}


Der vergleichende Blick auf Momente des Staunens im HE (B) und in den drei Geschichten aus 1001 Nacht ließ Analogien in Bezug auf ihre Auslöser und deren Folgen erkennen. Will man die Frage nach der Funktion des >Staunens/Wunderns < beantworten, so lässt sich konstatieren, dass der Einschub des Staunens/Wunderns meist einer Steigerung der Spannung auf narrativer Ebene, aber auch einer Retardation dient. Zudem fungiert das Staunen/Wundern auch als indirekte Hervorhebung der exklusiven Erlebnisse des Protagonisten. Schließlich werden über das Staunen Erinnerungen an erzählte Geschichten und ähnliche Erfahrungen evoziert, die ebenfalls aus dem Zustand des Unwissens in einen Zustand des Wissens, und zwar einem inter- bzw. transkulturellen Wissen, überführen und somit den Wissenshorizont über diesen Wissenstransfer um weitere Dimensionen erweitern.

Wissensaustausch und Horizonterweiterung fördern inter- und transkulturelle Kompetenzen und Praktiken, die aus der gemeinsamen Arbeit fachlicher und regionaler Expertisen an einem international und interdisziplinär ausgerichteten Interkulturalitätsprojekt ausgehandelt und entwickelt werden können. Eine solche wissenschaftliche Zusammenarbeit in Forschung und Lehre, auch wenn sie eine Umstrukturierung auf institutioneller Ebene erfordert, kann als gemeinsames Projekt das besondere Potential der Mediävistik um neue Perspektiven und um neue Möglichkeiten der Vermittelbarkeit erweitern und somit über den wissenschaftlichen Dialog hinaus auf humaner Ebene Empathie fördern.

\section{LITERATUR}

Amin, Samir (1999): Spectres of Capitalism. A Critique of Current Intellectual Fashions (1998). New Delhi (Nachdruck der Ausgabe New York).

Bein, Thomas (2010): Konrads von Megenberg "Buch der Natur" als germanistischmediävistisches Paradigma für einen innovativen interkulturellen akademischen Unterricht. In: Zeitschrift für interkulturelle Germanistik 1, H. 2, S. 25-47.

Bhatti, Anil (2015): Heterogenität, Homogenität, Ähnlichkeit. In: Zeitschrift für interkulturelle Germanistik 6, H. 1, S. 119-133.

Classen, Albrecht (2015): Transcultural Experiences in the Late Middle Ages: The German Literary Discourse on the Mediterranean World - Mirrors, Reflections, and Responses. In: Humanities 4, H. 4, S. 676-701.

Dembeck, Till (2017): Kulturdifferenz und sprachliche Vielfalt. In: Jeanne E. Glesener/ Nathalie Roelens / Heinz Sieburg (Hg.): Das Paradigma der Interkulturalität. Themen und Positionen in europäischen Literaturwissenschaften. Bielefeld, S. 9-19.

Die Geschichte des dritten Bettelmönches (1953). In: Die Erzählungen aus den Tausendundein Nächten. Vollst. dt. Ausg. in 6 Bde. zum ersten Mal nach d. arab. Urtext der Calcuttaer Ausg. aus d. Jahre 1830. Hg. u. übertr. v. Enno Littmann. Bd. I. Wiesbaden, S. 162-186. 
Die Geschichte des Prinzen von Karisme und der Prinzessin von Georgien (1926). In: Tausendundeine Nacht. Arabische Erzählungen. Dt. v. Maximilian Habicht, Friedrich Heinrich von der Hagen u. Carl Schall. Neu hg. v. Martin Schiller. Bd. 1. Leipzig, S. $140-169$.

Die Geschichte von Sindbad dem Seefahrer (1953). In: Die Erzählungen aus den Tausendundein Nächten. Vollst. dt. Ausg. in 6 Bde. zum ersten Mal nach d. arab. Urtext der Calcuttaer Ausg. aus d. Jahre 1830. Hg. u. übertr. v. Enno Littmann. Bd. IV. Wiesbaden, S. 97-201.

Eibach, Joachim / Opitz-Belakhal, Claudia/Juneja, Monica (2012): Kultur, Kulturtransfer und Grenzüberschreitungen. Joachim Eibach und Claudia Opitz im Gespräch mit Monica Juneja. In: zeitenblicke 11, Nr. 1 vom 7.11.2012; online unter: http://www. zeitenblicke.de/2012/1/Interview/index_html [Stand: 1.9.2018].

Geertz, Clifford ( ${ }^{31994): ~ D i c h t e ~ B e s c h r e i b u n g . ~ B e m e r k u n g e n ~ z u ~ e i n e r ~ d e u t e n d e n ~ T h e-~}$ orie von Kultur [1973]. In: Ders.: Dichte Beschreibung. Beiträge zum Verstehen kultureller Systeme. Übers. v. Brigitte Luchesi u. Rolf Bindemann. Frankfurt a.M., S. 7-43.

Gutjahr, Ortrud (2002): Alterität und Interkulturalität. Neuere deutsche Literatur. In: Claudia Benthien/Hans Rudolf Velten (Hg.): Germanistik als Kulturwissenschaft. Eine Einführung in neue Theorienkonzepte. Hamburg, S. 345-369.

Hahn, Alois (2000): Konstruktionen des Selbst, der Welt und der Geschichte. Aufsätze zur Kultursoziologie. Frankfurt a.M.

Ders./ Willems, Herbert (1999): Identität und Moderne. Frankfurt a.M.

Heimböckel, Dieter (2015): Im Grenzgang. Für eine Germanistik als Schwellenkunde. Vortrag gehalten auf der Tagung des italienischen Germanistenverbandes (AIG): Reti internazionali e prospettive della ricerca germanistica/Internationale Netzwerke und Perspektiven der germanistischen Forschung (Istituto Italiano di Studi Germanici, Villa Sciarra-Wurts, Rom, 3.-4. Oktober 2014). In: Zeitschrift für interkulturelle Germanistik 6, H. 1, S. 151-161.

Ders./ Weinberg, Manfred (2014): Interkulturalität als Projekt. In: Zeitschrift für interkulturelle Germanistik 5, H. 2, S. 119-143.

Herder, Johann Gottfried (1891): Auch eine Philosophie der Geschichte zur Bildung der Menschheit. In: Ders.: Sämtliche Werke. Hg. v. Bernhard Suphan. Bd. 5. Berlin, S. 475-586.

Herweg, Mathias (2017): Alterität und Kontinuität. Vom interkulturellen Potential der germanistischen Mediävistik. In: Zeitschrift für interkulturelle Germanistik 8, H. 1, S. 11-23.

Herzog Ernst. Ein mittelalterliches Abenteuerbuch (1972). In der mittelhochdeutschen Fassung B nach der Ausg. v. Karl Bartsch mit den Bruchstücken der Fassung A. Hg., übers., mit Anmerkungen u. einem Vorwort versehen v. Bernhard Sowinsiki. Stuttgart.

Hofmann, Michael (2006): Interkulturelle Literaturwissenschaft: Eine Einführung. Paderborn.

Iljassova-Morger, Olga (2009): Transkulturalität als Herausforderung für die Literaturwissenschaft und Literaturdidaktik. In: Das Wort. Germanistisches Jahrbuch. Russland, S. 37-57. 
Joyce, James (2000): Ireland, Island of Saints and Sages. In: Kevin Barry (Hg.): James Joyce. Occasional, Critical, and Political Writing. Neu-Delhi, S. 108-127.

Leggewie, Claus/Zifonun, Dariuš (2010): Was heißt Interkulturalität? In: Zeitschrift für interkulturelle Germanistik 1, H. 1, S. 11-31.

Martinson, Steven D. (2008): Transcultural German Studies: Theorie und Literaturinterpretation. In: Ders./Renate A. Schulz (Hg.): Transcultural German Studies. Bern/Berlin, S. 73-84.

Mersch, Margit (2016): Transkulturalität, Verflechtung, Hybridisierung - ıneue، epistemologische Modelle in der Mittelalterforschung. In: Wolfram Drews/Christian Scholl (Hg.): Transkulturelle Verflechtungsprozesse in der Vormoderne. Berlin, S. 239-251; online unter: https://ruhr-uni-bochum.academia.edu/MargitMersch [Stand: 1.9.2018].

Mecklenburg, Norbert (2008): Das Mädchen aus der Fremde. Germanistik als interkulturelle Literaturwissenschaft. München.

Netzwerk Transkulturelle Verflechtungen (Hg.; 2016): Transkulturelle Verflechtungen: mediävistische Perspektiven. Kollaborativ verf. v. Georg Christ u.a. Göttingen.

Noll, Frank Jasper (2015): Tagungsbericht: Enzyklopädisches Erzählen und vormoderne Romanpoetik (1400-1700). 14.10.2015-16.10.2015, Wolfenbüttel. In: H-Soz-Kult, 23.12.2015; online unter: http://www.hsozkult.de/conferencereport/id/tagungs berichte-6314 [Stand: 1.9.2018].

Pries, Ludger (2000): "Transmigranten " als ein Typ von Arbeitswanderern in pluri-lokaIen sozialen Räumen. In: Ingrid Gogolin/Bernhard Nauck (Hg.): Migration, gesellschaftliche Differenzierung und Bildung. Resultate des Forschungsschwerpunktprogramms FABER. Wiesbaden, S. 415-437.

Quenstedt, Falk (2017): The Marvellous in Transfer: Sindbad, the Sailor (as-Sindibād al-baḥrī) and Different Versions of Herzog Ernst - Vortrag im Rahmen der internationalen Tagung Medieval Texts in Transit. Freie Universität Berlin, 22. Juli 2017.

Ders. (2018): [Art.] "Indien. Mirabilienorient". In: Tilo Renz/Monika Hanauska/ Mathias Herweg (Hg.): Literarische Orte in deutschsprachigen Erzählungen des Mittelalters. Ein Handbuch. Berlin/ Boston.

Renz, Tilo/Hanauska, Monika/Herweg, Mathias (2018): Einleitung. In: Dies. (Hg.): Literarische Orte in deutschsprachigen Erzählungen des Mittelalters. Ein Handbuch. Berlin/ Boston, S. 1-11.

Rieger, Stefan/Schahadat, Schamma/Weinberg, Manfred (1999): Interkulturalität zwischen Inszenierung und Archiv. Vorwort. In: Dies. (Hg.): Interkulturalität. Zwischen Inszenierung und Archiv. Tübingen, S. 9-26.

Salama, Dina Aboul Fotouh Hussein (2003): Das Orientbild im Herzog Ernst zwischen Wirklichkeit und Phantastik. Diss. (masch. ) Universität Kairo.

Dies. (2015): Die literarische Imagologie dunkelhäutiger Frauen in Strickers "Königin von Mohrenland“ (zw. 1210-1230). In: Zeitschrift für interkulturelle Germanistik 6, H. 1, S. 9-29.

Dies. (2016): Transkulturalität im Tristan Gottfrieds von Straßburg. Verflechtung und Entflechtung als Konzept der Identität. In: Archiv für das Studium der Sprachen und neueren Literaturen, 253. Bd., H. 1, S. 1-20. 
Dies. (2018): Mediävistik in Ägypten. Ein Essay. In: Jahrbuch für Internationale Germanistik (im Erscheinen).

Schlesier, Renate (1996): Das Staunen ist der Anfang der Anthropologie. In: Hartmut Böhme/Klaus R. Scherpe (Hg.): Literatur und Kulturwissenschaften. Positionen, Theorien, Modelle. Reinbek b. Hamburg, S. 47-59.

Schöning, Udo (2000): Die Internationalität nationaler Literaturen. Bemerkungen zur Problematik und ein Vorschlag. In: Ders. (Hg.): Die Internationalität nationaler Literaturen. Beiträge zum Ersten Symposion des Göttinger Sonderforschungsbereichs 529. Göttingen, S. 9-43.

Sieburg, Hans (2015/2016): Interkulturelle Mediävistik als aktuelle Herausforderung germanistischer Kulturwissenschaft? In: Kairoer Germanistische Studien 22, Kongressakten, S. 393-400.

Sivri, Yücel (2016): Mitteldeutsche Orientliteratur des 12. und 13. Jahrhunderts. "Graf Rudolf» und "Herzog Ernst». Ein Beitrag zu interkulturellen Auseinandersetzungen im Hochmittelalter. Frankfurt a.M.

Spuler, Richard (1983): The Orientreise of Herzog Ernst. In: Neophilologus 67, S. 410418.

Szklenar, Hans (1966): Studien zum Bild des Orients in vorhöfischen deutschen Epen. Göttingen.

Trommsdorf, Gisela (2000): Internationale Kultur? Kulturpsychologische Aspekte der Globalisierung. In: Ingrid Gogolin/ Bernhard Nauck (Hg.): Migration, gesellschaftliche Differenzierung und Bildung. Wiesbaden, S. 387-414.

Dies./Friedlmeier, Wolfgang (2004): Zum Verhältnis zwischen Kultur und Individuum aus der Perspektive der kulturvergleichenen Psychologie. In: Aleida Assmann u.a. (Hg.): Positionen der Kulturanthropologie. Frankfurt a.M., S. 358-386.

Tugendhat, Ernst (2004): Egozentrität und Mystik. Eine anthropologische Studie. München.

Welsch, Wolfgang (2010): Was ist eigentlich Transkulturalität? In: Lucyna Darowska/ Thomas Luttenberg/Claudia Machold (Hg.): Hochschule als transkultureller Raum? Kultur, Bildung und Differenz in der Universität, Bielefeld, S. 39-66.

Wittgenstein, Ludwig (1975): Philosophische Untersuchungen. Frankfurt a.M. 